\title{
Photosynthèse d'une culture en rangs de tomates sous serre. Modélisation analytique et cartographie de l'activité du feuillage
}

\author{
M Tchamitchian, JJ Longuenesse
}

avec la collaboration technique de B Brunel et C Sarrouy

INRA, station de bioclimatologie, domaine Saint-Paul, BP 91, 84143 Montfavet cedex, France

(Reçu le 15 mai 1990; accepté le 12 novembre 1990)

\begin{abstract}
Résumé - Afin de fournir les éléments d'une optimisation dynamique de la régulation des différents paramètres climatiques sous serre, un modèle mécaniste du fonctionnement d'un couvert en rangs a été élaboré sur la base du modèle d'interception du rayonnement solaire de Sinoquet (1989), puis calé et validé sur une culture de tomates de printemps. A partir de la connaissance du rayonnement incident au sommet de la culture, de sa répartition en ses composantes directe et diffuse et de la position du soleil, il calcule l'interception du rayonnement en tenant compte des rediffusions, ainsi que de l'activité photosynthétique du couvert.

Deux fonctions de réponse photosynthétique foliaire ont été utilisées :

- une fonction hyperbolique classique caractérisant les potentialités moyennes de l'ensemble des feuilles de la culture (modèle 1);

- une fonction tenant compte de l'âge des feuilles, leurs capacités photosynthétiques étant d'autant plus faibles qu'elles sont âgées (modèle 2).

Le calage a été réalisé à partir de mesures de photosynthèse foliaire, la validation avec des mesures in situ de la photosynthèse de plantes entières. Les mesures nécessaires au fonctionnement du modèle (rayonnement, description géométrique de la surface foliaire) ont été faites sur la même culture.

La comparaison des simulations et des mesures de photosynthèse du couvert montre une assez bonne adéquation des 2 modèles. Cependant, le modèle avec effet âge sous-estime l'activité du couvert, lorsqu'il est soumis à un fort rayonnement; ce biais peut être attribué à une sous-estimation des capacités photosynthétiques des feuilles âgées. Nous avons simulé l'expérience présentée par Acock et al (1978) où l'activité de couches de feuilles d'âges différents a été mesurée par effeuillages successifs. Le modèle 2, avec effet âge, conduit à une photosynthèse équivalente, mais répartie différemment, essentiellement réalisée par la couche supérieure. La sous-estimation des capacités photosynthétiques des feuilles âgées apparaît nettement.
\end{abstract}

photosynthèse / modèle / rayonnement solaire intercepté / culture en rangs / serre / tomate

Summary - Photosynthesis of a greenhouse row crop of tomatoes. Development of an analytical model and mapping of foliage activity. On the basis of the solar radiation interception model of Sinoquet (1989), we developed a mechanistic model of the photosynthesis of a row crop, to be used for the dynamic optimization of greenhouse climate. This model was calibrated and validated on a spring tomato crop.

The canopy is divided into units of assumed uniform leaf area density, leaf angle distribution and photosynthetic PAR response. This division can be made along 2 or 3 axes, thus allowing variability along the rows to be accounted for the rows as well as across them (fig 1). From the input of incident diffuse and direct radiation on top of the canopy, the model computes, for each canopy unit, the leaf area distribution with respect to PAR interception, taking into account multiple diffusions, and photosynthetic activity. The canopy carbon gain is then computed as the sum of the unit values.

Two leaf photosynthetic light-response functions were used:

- one single hyperbolic function to describe the mean activity of all the leaves of the crop (model 1);

- one function which includes a reduction factor proportional to leaf age, with the lowest photosynthetic rate for the oldest leaves.

Measures of leaf photosynthesis were used to calibrate the model; the validation was made with in situ measures of whole-plant $\mathrm{CO}_{2}$ exchanges done under different radiative conditions (fig 2).

Incident irradiance and canopy structure measurements were also performed on the same crop, and used in the model.

Comparing in situ measures of crop photosynthesis with simulated data shows a good predictive value of both models, although the model with a leaf age effect under-estimates crop photosynthesis under high irradiance (fig 3).

We analyzed the interaction between the date and hour of photosynthesis measurement on the residuals of the mo- 
dels (fig 4) and we compared the simulations performed with the two models (fig 5). From this analysis, we concluded that the under estimation of crop assimilation by model 2 is mainly due to a too high reduction factor for the oldest leaves.

We simulated the experiment in which Acock et al (1978) measured the photosynthetic activity of canopy layers of different leaf age by successive partial defoliations (fig 6). Model 2, including a leaf age effect, computes a value of crop photosynthesis similar to that mesured by Acock et al (1978) but the distribution among the crop layers is distorted; the under-estimation of the activity of the older leaves is clearly shown.

photosynthesis / model / intercepted solar radiation / row canopy / greenhouse / tomato

\section{INTRODUCTION}

Les progrès réalisés dans la modélisation du fonctionnement physique des serres, et le développement de l'informatique pour leur pilotage, permettent, à l'heure actuelle, une régulation beaucoup plus fine que par le passé des différents paramètres du climat.

L'optimisation dynamique, à court et à long terme, de ces paramètres en fonction d'objectifs agronomiques ou économiques exige une connaissance fine de leur influence sur le fonctionnement des plantes, et en particulier sur les éléments du bilan de carbone : la régulation du climat sur des bases physiologiques suppose, en particulier, une modélisation de la photosynthèse, composante essentielle de ce bilan, à un pas de temps court (inférieur à l'heure) et aussi une grande résolution spatiale.

II est alors possible d'explorer différents modes de conduite (densité, contre-plantation, disposition en rangs simples ou doubles, etc.) et de préparer ainsi une expérimentation portant sur ces modes de conduite.

Dans la plupart des modèles de photosynthèse d'un couvert en rangs, la végétation occupe un volume de forme ou de section connue. Lorsque la végétation est supposée uniforme le long de l'axe des rangs, la section du volume est elliptique (Charles-Edwards et Thorpe, 1976) ou plus communément triangulaire ou rectangulaire (Allen, 1974; Jackson et Palmer, 1979; Gijzen, Goudriaan, 1989). Quelques auteurs, définissant le rang comme une succession de plantes, ont décrit le volume occupé par une plante (Thorpe et al, 1978; Whitfield, 1986), ce qui se justifie pleinement dans le cas des vergers. Dans tous les cas, la végétation, dans ces volumes, est supposée homogène horizontalement, et même souvent verticalement. A l'intérieur du volume, l'absorption du rayonnement est calculée en utilisant le formalisme des modèles probabilistes au sens où Lemeur et Blad (1974) l'entendent, mais en faisant souvent de nombreuses hypothèses réductrices.
Ces modèles dépendent étroitement du couvert auxquels ils s'appliquent, en raison de la description géométrique des limites de ce couvert. En plus des hypothèses faites sur l'homogénéité de la culture, ils négligent généralement les réflexions et les rediffusions du rayonnement à l'intérieur de la culture.

D'autres auteurs, beaucoup moins nombreux, ont adopté une approche où les limites de l'espace occupé par la végétation ne sont plus décrites (Iwakiri et Inayama, 1974; Cohen et Fuchs, 1987). L'espace est découpé en couches horizontales et en tranches verticales. Leurs intersections définissent des «tubes» à section rectangulaire (le rang est supposé homogène dans sa longueur). Tous ces éléments sont caractérisés par une densité foliaire, homogène dans chaque tube, mais qui peut être nulle. L'absorption du rayonnement et la distribution des surfaces foliaires par rapport à ce rayonnement est alors calculée pour chaque tube. Cette méthode peut être utilisée pour des couverts de formes très différentes.

Une fonction de réponse photosynthétique de l'unité de surface foliaire au rayonnement incident transforme alors l'énergie reçue en photosynthèse. Ces réponses, pondérées par la distribution de surface foliaire donnent accès à la photosynthèse du couvert. Ces fonctions de réponse sont toujours, à notre connaissance, considérées identiques pour l'ensemble de la surface foliaire d'un couvert.

Dans le cas des cultures en rangs, les feuilles âgées situées à la base du couvert reçoivent, pour certaines hauteurs solaires, un éclairement important. Ne pas prendre en compte la variation des capacités photosynthétiques des feuilles en fonction de leur âge peut donc conduire à surestimer la photosynthèse instantanée du couvert.

Le modèle que nous proposons ici décrit de façon instantanée l'interception du rayonnement et la photosynthèse d'une culture en rangs sous serre. Il s'appuie sur un modèle probabiliste d'interception des rayonnements solaires par une culture en rangs (Sinoquet, 1989). Les mesures 
utilisées pour le calage et la validation du module rayonnement comme du module photosynthèse ont été réalisées sur une culture de tomates de printemps sous serre. Nous avons aussi simulé les situations présentées par Acock et al (1978), leur protocole de mesure permettant d'isoler les activités de feuilles de différents âges.

\section{MATÉRIELS ET MÉTHODES}

\section{Méthode}

\section{Présentation du modèle}

Le modèle d'échanges radiatifs (Sinoquet, 1989) que nous avons utilisé en le modifiant, calcule le rayonnement moyen intercepté par chaque cellule du système. Ces cellules sont définies par l'intersection de couches horizontales et de tranches verticales pour l'espace occupé par la végétation, par les bandes de sol qui limitent chacune des tranches, et enfin par le ciel (fig 1). Chaque cellule de l'espace occupé par la culture est caractérisée par sa densité foliaire (qui peut être nulle entre les rangs), la distribution d'inclinaison des éléments de feuillage et les propriétés optiques de ces éléments. Les bandes de sol sont caractérisées par leur réflectance. Le ciel est défini par sa luminance (il est, avec le soleil, la seule source de rayonnement dans le domaine PAR qui nous intéresse).

A partir de cette caractérisation et de paramètres complémentaires calculés, le modèle calcule le rayonnement intercepté par chaque cellule de végétation et chaque bande de sol (cf Sinoquet, 1989, pour le détail de la démarche).

Cette information est toutefois insuffisante pour effectuer un calcul de photosynthèse. Compte tenu de la non linéarité de la réponse photosynthétique au rayonnement, il faut une description des couples (surface foliaire-rayonnement intercepté). De plus, la fonction de photosynthèse foliaire que nous avons utilisée se ré-

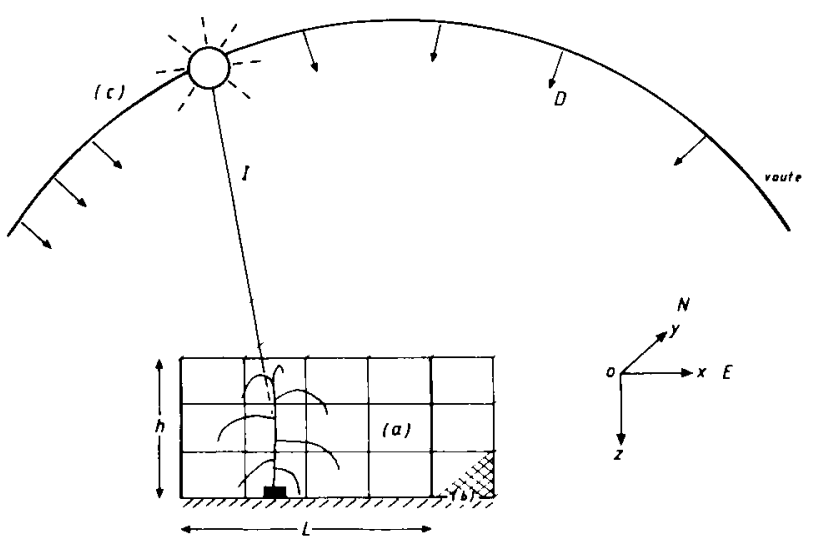

Fig 1. Le système modélisé: a : culture, de hauteur $h$ et de distance inter-rang $L$ divisée en cellules de section rectangulaire et de longueur infinie; $\mathbf{b}:$ sol; $\mathbf{c}$ : voûte céleste; $D$ : rayonnement diffus; $I$ : rayonnement solaire direct. fère à un rayonnement descendant, le rayonnement rediffusé éclairant la face inférieure des feuilles étant ignoré.

Séparant les surfaces ensoleillées de celles qui sont à l'ombre, nous affectons à ces dernières la valeur de ce rayonnement diffus descendant qu'elles interceptent, qu'il soit directement d'origine solaire ou rediffusé par le feuillage (désormais appelé diffus descendant par opposition au rayonnement diffus solaire). Ce rayonnement diffus descendant intercepté est supposé uniforme au sein de chaque cellule. Pour les surfaces ensoleillées, il faut prendre en compte les variations de l'angle d'incidence du rayonnement direct dues aux diverses positions que prennent les feuilles dans l'espace.

Seul le domaine PAR $(400-700 \mathrm{~nm})$ sera considéré ici, puisque seul utile à la photosynthèse.

\section{Répartition des surfaces foliaires ensoleillées et ombrées}

Le calcul de la surface foliaire ensoleillée se fait de façon classique (Lemeur et Blad, 1974) :

$$
S^{e}=\left(1-P_{0}\right) / K(\Omega)
$$

où $S^{e}$ est la surface ensoleillée, $P_{0}$ la fréquence de trous cumulée et $K(\Omega)$ le coefficient d'extinction pour l'épaisseur de feuillage considérée lorsque l'agrégation foliaire est aléatoire. Ce calcul peut être détaillé par inclinaison et par cellule :

$$
S_{c, \alpha}^{e}=P_{0}^{\sup _{0}}\left[\left(1-P_{0, c}\right) / K(\Omega) g(\alpha)\right]
$$

ou $S_{c, \alpha}^{e}$ est la surface ensoleillée d'inclinaison $\alpha$ dans la cellule $c, g(\alpha)$ la distribution d'inclinaison des sur-

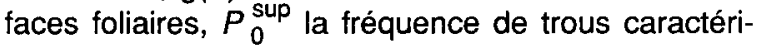
sant la végétation située au-dessus de la cellule considérée, $P_{0, c}$ la fréquence de trouée de la cellule (c) pour laquelle on fait le calcul.

La surface ombrée $S_{c}^{\circ}$ se calcule par différence entre la surface totale de la cellule et la surface ensoleillée.

\section{Introduction du calcul de photosynthèse}

Pour chacune des situations identifiées lors de l'étape précédente par des couples (surface-rayonnement intercepté), nous effectuons un calcul d'activité photosynthétique. La somme de ces réponses, pondérée par les surfaces foliaires donne l'activité de la cellule considérée :

$$
P_{c}^{\circ}=S_{c}^{0} f\left(D_{c}\right)+\sum_{\alpha=0}^{\pi / 2}\left[\sum_{\phi=0}^{2 \pi}\left[S_{c, \alpha, \phi}^{e} f\left(D_{c}+l_{c, \alpha, \phi}\right)\right]\right]
$$

où $f$ est la fonction de calcul de la photosynthèse par unité de surface foliaire, $D$ le rayonnement diffus descendant intercepté par la cellule $c$ et / le rayonnement direct incident sur la surface foliaire d'inclinaison $\alpha$ et d'azimut $\phi$. Le modèle utilisé suppose une distribution 
uniforme des azimuts mais peut tenir compte d'une distribution des inclinaisons foliaires.

La photosynthèse de la plante entière se définit naturellement comme la somme des $P_{c}$ :

$$
P=\sum_{c} P_{c}
$$

\section{Description de la photosynthèse des feuilles}

La photosynthèse de l'unité de surface de feuille en fonction du rayonnement est décrite par une fonction hyperbolique classique, du type de celle utilisée par exemple par Chartier (1969), Van Bavel (1975), Acock et al (1976) :

$$
P_{b}=\frac{P_{\max } l}{P_{\max } / E_{i}+l}
$$

$P_{b}$ : photosynthèse brute;

$P_{\text {max }}$ : photosynthèse brute maximale à 350 ppm; $E_{i}$ : efficience quantique maximale à $350 \mathrm{ppm}$;

$I$ : éclairement quantique incident.

Cette fonction peut être éventuellement modulée par un facteur de prise en compte de l'effet de l'âge de la feuille, décrit ici par une décroissance linéaire au-delà d'un âge-seuil. Cet âge est mesuré par un index correspondant au rang de la feuille sur la tige, en comptant à partir de la première feuille de plus de $1 \mathrm{~cm}$ de long.

On écrira alors :

$$
P_{b 0}=\frac{P_{\max 0} I}{P_{\max 0} / E_{i 0}+l}
$$

où les coefficients $P_{\max 0}$ et $E_{i 0}$ sont caractéristiques des feuilles supérieures de la plante (les plus jeunes et les mieux éclairées), et la photosynthèse d'une feuille de rang $P /$ s'écrira:

$$
P_{b P l}=P_{b o} F_{P I}
$$

avec

$$
\begin{array}{ll}
F_{P I}=1 & \text { si } P I<P I_{0} ; \\
F_{P I}=1-\frac{P I-P I_{0}}{P I_{\mathrm{m}}-P I_{0}} & \text { si } P I_{0} \leq P I \leq P I_{\mathrm{m}} ; \\
F_{P I}=0 & \text { si } P I>P I_{\mathrm{m}}
\end{array}
$$

$P I:$ âge de la feuille

$P l_{0}$ : seuil de début de réduction de la photosynthèse $P I_{m}$ : âge au-delà duquel on considère l'assimilation comme négligeable.

Nous avons introduit dans le modèle global deux jeux de coefficients :

- des valeurs moyennes pour l'ensemble des feuilles du couvert (correspondant à l'équation 1) :

$P_{\text {max }}=1,40 \mathrm{mg} \mathrm{CO} \cdot \mathrm{m}^{-2 \cdot \mathrm{s}^{-1}}$

$E_{i}=0,0016 \mathrm{mg} \mathrm{CO}_{2} \cdot \mu_{\mathrm{mol}}^{-1}$
- des valeurs prenant en compte le gradient lié à l'âge (correspondant à l'équation 2):

$$
\begin{array}{ll}
P_{\max 0} & =2,01 \mathrm{mg} \mathrm{CO}{ }_{2} \cdot \mathrm{m}^{-2} \cdot \mathrm{s}^{-1} \\
E_{i 0} & =0,0025 \mathrm{mg} \mathrm{CO}_{2} \cdot \mu \mathrm{mol}^{-1} \\
P I_{0} & =9 \\
P I_{\mathrm{m}} & =39
\end{array}
$$

\section{Protocole expérimental}

Le modèle a été validé par des mesures de photosynthèse à l'échelle de la plante entière, réalisées sur une culture de printemps sous serre verre.

\section{Matériel végétal et mode de culture}

Les tomates (cultivar Duranto), cultivées en hors-sol sur substrat de pouzzolane, étaient disposées en simples rangs, orientés nord-sud, espacés de $1 \mathrm{~m}$, avec une plante tous les $0,4 \mathrm{~m}$ sur le rang, soit une densité de 2,5 plantes $\cdot \mathrm{m}^{-2}$. La serre utilisée est une unité de $6 \times 30 \mathrm{~m}$, de grand axe orienté nord-sud, enclavée dans un ensemble de 4 unités semblables plus 2 éléments de bordure. Elle est chauffée par 3 aérothermes basse température, et ventilée par 4 extracteurs placés dans le pignon sud, et des ouvrants côté nord. La régulation climatique est de type classique par thermostat (consignes $15^{\circ} \mathrm{C}$ pour le chauffage et $25^{\circ} \mathrm{C}$ pour la ventilation). L'irrigation fertilisante au goutte à goutte est pilotée par un automate climatique (Meteodyn).

Les tomates ont été semées le 2 mars 1989 en pépinière sur godets de laine de roche, et mises en place dans la serre le 15 avril 1989. Les mesures ont commencé le 20 mai 1989 et ont duré jusqu'au 4 juillet 1989.

\section{Caractérisation du LAI et de la géométrie de la culture}

La surface foliaire, ses distributions spatiale et d'orientation ont été mesurées à l'aide d'une méthode adaptée de la méthode des silhouettes (Bonhomme et Varlet-Grancher, 1978). Une maquette métallique représentant la tige et les nervures principales des feuilles est réalisée. Cela permet de placer les "feuilles" métalliques dans un même plan pour permettre le traitement de cette maquette par la méthode des silhouettes (Tchamitchian, 1990).

La distribution observée des azimuts foliaires n'est pas significativement différente de la distribution uniforme que suppose le modèle d'interception du rayonnement.

Le LAl de la culture était de 2,5 au début de la période de mesure de la photosynthèse du couvert, de 2,8 à la fin. La distribution d'inclinaison des feuilles est restée relativement constante durant cette période, les inclinaisons les plus représentées étant d'environ $45^{\circ}$ (Tchamitchian, 1990). 


\section{Mesure des rayonnements solaires}

Le rayonnement $P A R$ incident total était mesuré au sommet de l'enceinte de mesure des échanges gazeux, sous la couverture plastique de cette enceinte. Des relations entre les composantes directes et diffuses à l'extérieur et à l'intérieur de la serre ont permis la détermination des valeurs du rayonnement $P A R$ direct et PAR diffus incident (Tchamitchian, 1990).

\section{Mesure des échanges de $\mathrm{CO}_{2}$}

Avant la mise en place de la culture, des bacs métalliques sans fond, en tôle galvanisée, d'une dimension de 0,6 $61,6 \mathrm{~m}$, sont disposés en plusieurs endroits de la serre, à cheval sur un rang. Les bords de ces bacs s'enfoncent dans le sol de 0,05 m environ. Aux périodes de mesure, ces bacs servent de support à des cadres sur lesquels on ajuste une enveloppe de film plastique (polyéthylène $30 \mu \mathrm{m}$ ). Quatre plantes sont ainsi isolées dans une enceinte.

Ce système en circuit ouvert permet un suivi en continu des échanges gazeux des plantes qui s'y trouvent (photosynthèse, respiration, transpiration). II est piloté, par l'intermédiaire d'un module d'acquisition et de contrôle (Analog Devices Micromac 4000), par micro-ordinateur compatible IBM-PC qui contrôle le cycle de mesure et effectue les calculs de base (Daudet, 1987).

Ce dispositif est maintenu en place sur un bac pendant 10-15 j; il est ensuite déplacé sur un autre bac, de façon à éviter de perturber significativement le fonctionnement des plantes étudiées.

Les mesures fournies par notre dispositif sont des échanges nets de $\mathrm{CO}_{2}$ de l'ensemble formé par les plantes et leur substrat de culture; dans l'impossibilité de séparer la part de respiration attribuable d'une part aux feuilles, d'autre part aux tiges et aux substrats (racines et micro-organismes), et de modéliser cette dernière, nous avons ramené ces résultats à des valeurs de photosynthèse brute (photosynthèse nette + respiration obscure) en calculant une valeur moyenne de pertes respiratoires pendant la nuit, et en lui appliquant pendant la journée une correction de température avec un $Q_{10}$ de 1,4 (Gary, 1989); les pertes respi-

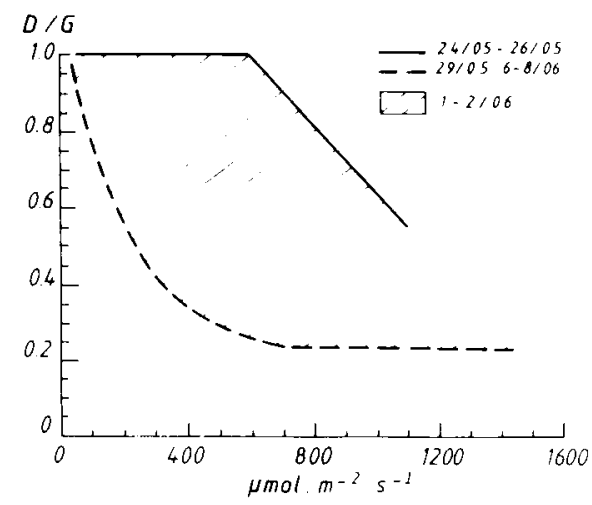

Fig 2. Evolution du rapport Diffus/Global en fonction du $P A R$ total $\left(\mu \mathrm{mol} \cdot \mathrm{m}^{-2} \cdot \mathrm{s}^{-1}\right)$ au cours des journées utilisées pour la validation. ratoires ainsi calculées sont ajoutées aux échanges mesurés pour fournir une valeur de photosynthèse brute calculée.

\section{Choix des données utilisées pour la validation}

Par beau temps, lorsque l'ombre d'un élément structurel de la serre (faîtière, chéneau, petit bois, etc.) passe sur la capteur $P A R$, celui-ci enregistre une baisse de rayonnement plus importante que celle subie par l'ensemble de l'enceinte. Inversement, une partie du feuillage des plantes peut se trouver dans l'ombre sans que le capteur soit affecté. Les périodes correspondantes doivent donc être éliminées de la relation entre rayonnement incident et échanges gazeux.

Par temps couvert, en revanche, ce phénomène est négligeable puisque la totalité du rayonnement incident est reçue sous forme de rayonnement diffus que l'on peut considérer en première approximation comme constant sur l'ensemble de la surface considérée.

Nous avons sélectionné pour la validation du modèle, 7 journées entre le 24 mai et le 8 juin, représentant différents types de temps : belle journée à nébulosité nulle, alternance de passages nuageux et d'éclaircies, périodes couvertes (fig 2).

Le 24 et le 26 mai sont des journées à dominante de temps couvert, au cours desquelles le rapport $D / G$ (rayonnement diffus sur rayonnement total) reste proche de 1 même pour des valeurs de rayonnement relativement élevées; ce n'est que vers le milieu de la journée qu'il diminue légèrement, sans jamais tomber en-dessous de 0,5 (classe 1 );

Le 29 mai, le 6 et le 8 juin sont des journées à dominante de ciel clair au cours desquelles la valeur de $\mathrm{D} / \mathrm{G}$ diminue très régulièrement dès le début de la journée et se stabilise aux alentours de 0,25 (classe 2);

Le 1 et le 2 juin, enfin, sont caractérisées par un temps très variable et des valeurs de $D / G$ qui varient sans cesse entre les limites représentées par les deux situations précédentes (classe 3 ).

\section{Validation du modèle}

Les valeurs brutes de photosynthèse calculées ont été comparées aux valeurs simulées par le modèle. Ces comparaisons ont été réalisées sous les 2 hypothèses envisagées page 20 :

- une photosynthèse foliaire homogène sur l'ensemble de la culture, sans influence de l'âge ni de la position des feuilles (modèle 1);

- une décroissance régulière de la photosynthèse en fonction du rang de la feuille compté à partir du sommet de la plante (modèle 2). 


\section{Qualité globale du modèle}

Sur l'ensemble des journées de mesures que nous avons étudiées, le coefficient d'ajustement est de 0,97 pour le modèle 1 et de 0,94 pour le modèle 2; les valeurs des résidus sont généralement négatives (médianes respectivement de -17 et $-53 \mathrm{mg}$ de $\mathrm{CO}_{2} \cdot \mathrm{m}^{-2} \cdot \mathrm{s}^{-1}$ avec une dispersion relativement importante (écart type des résidus de l'ordre de 100, et erreur standard de l'ordre de $4 \mathrm{mg}$ de $\mathrm{CO}_{2} \cdot \mathrm{m}^{-2} \cdot \mathrm{s}^{-1}$ ) (fig 3).

On obtient globalement une précision satisfaisante de la description du fonctionnement du couvert, mais avec une tendance à sous-estimer la photosynthèse, particulièrement lorsqu'on tient compte du facteur de vieillissement des feuilles.

Le modèle étant très sensible à la valeur du rapport $D / G$, il est possible qu'un biais systématique, lié à une erreur dans la détermination de ce rapport, apparaisse sur les photosynthèses simulées.

Nous avons donc voulu vérifier si la classification en fonction du type de temps se retrouvait dans les résidus du modèle.

L'analyse de variance des résidus (tableau I) ne fait apparaître aucune différence entre les journées des classes 1 et 2 . L'augmentation des résidus pour les journées de la classe 3 est probablement liée à la grande variabilité du rayonnement d'un instant à

Tableau I. Analyse de variance des résidus du modèle 1 en fonction du type de temps (résidus en $\mathrm{mg}$ $\mathrm{CO}_{2} \cdot \mathrm{m}^{-2}$ de sol-s ${ }^{-1}$ ). 1 : journées essentiellement couvertes; 2 : journées très dégagées; 3 : journées de rapport $D / G$ variable.

\begin{tabular}{cccc}
$\begin{array}{c}\text { Type de } \\
\text { temps }\end{array}$ & Moyenne & $\begin{array}{c}\text { Intervalle de confiance } \\
\text { autour de la moyenne } \\
\text { (seuil 5\%) }\end{array}$ \\
\hline & & & 0 \\
2 & $-13,7$ & -27 & -11 \\
3 & $-18,0$ & -25 & -28 \\
\hline
\end{tabular}

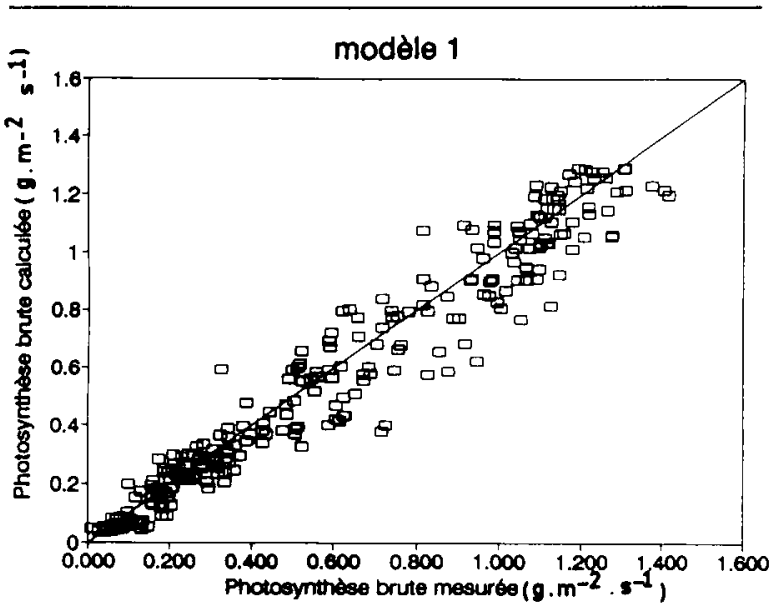

l'autre, et aux erreurs qu'elle entraîne lors du calcul du rayonnement diffus par interpolation entre 2 instants de mesure à l'extérieur.

De ce point de vue, le fonctionnement du modèle est donc satisfaisant, et la variabilité observée est plus liée à des problèmes de conditions expérimentales qu'à ses défauts intrinsèques.

\section{Analyse détaillée sur les résidus du modèle 1 (sans effet âge)}

La caractéristique essentielle des cultures en rangs que ce modèle permet de prendre en compte est la grande variabilité de la répartition du rayonnement au sein du couvert en fonction de la hauteur du soleil, et donc de l'heure de la journée, ou plus exactement, dans notre cas, l'écart au midi solaire, puisque notre culture est orientée nord-sud, et que la course apparente du soleil est donc parfaitement symétrique par rapport aux rangs.

Nous avons donc réalisé une analyse plus fine des résidus du modèle en fonction de ce paramètre (tableau (I).

Tableau II. Analyse des résidus (mg $\mathrm{CO}_{2} \cdot \mathrm{m}^{-2}$ de sol. $\mathrm{s}^{-1}$ ) des modèles 1 et 2 en fonction de l'écart au midi solaire (classes de $2 \mathrm{~h}$ ).

\begin{tabular}{cccc}
\hline $\begin{array}{c}\text { Ecart au } \\
\text { midi solaire }\end{array}$ & Moyenne & $\begin{array}{c}\text { Intervalle de confiance } \\
\text { autour de la moyenne } \\
\text { (seuil 5\%) }\end{array}$ \\
\hline & & \\
\hline Modèle 1, sans effet âge. & \\
$0-2 \mathrm{~h}$ & $-56,8$ & -72 & -42 \\
$2-4 \mathrm{~h}$ & $-23,0$ & -35 & -11 \\
$4-6 \mathrm{~h}$ & $-14,5$ & -28 & -1 \\
$>6 \mathrm{~h}$ & $-10,8$ & -25 & 3 \\
Modèle 2, avec effet âge. & & \\
$0-2 \mathrm{~h}$ & $-144,0$ & -161 & -127 \\
$2-4 \mathrm{~h}$ & $-128,6$ & -142 & -115 \\
$4-6 \mathrm{~h}$ & $-25,5$ & -40 & -11 \\
$>6 \mathrm{~h}$ & $-6,5$ & -23 & 8 \\
\hline
\end{tabular}

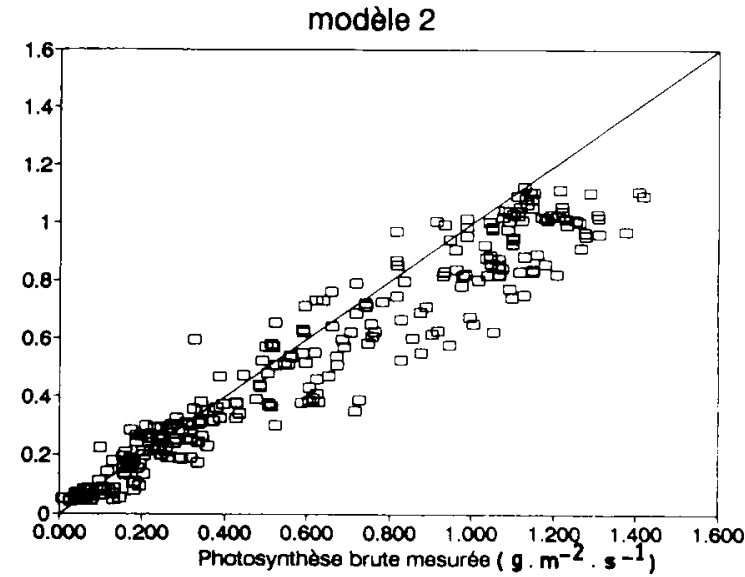

Fig 3. Relation entre photosynthèse mesurée et simulée par les 2 modèles (en $\mathrm{mg}$ de $\mathrm{CO}_{2} \cdot \mathrm{m}^{-2}$ de sol•s $\mathrm{s}^{-1}$ ): $\mathbf{a}$ : modèle 1 , sans effet âge; b: modèle 2, avec introduction d'un effet réducteur de l'âge des feuilles sur leurs capacités photosynthétiques. 
a moyenne des résidus du modèle 1

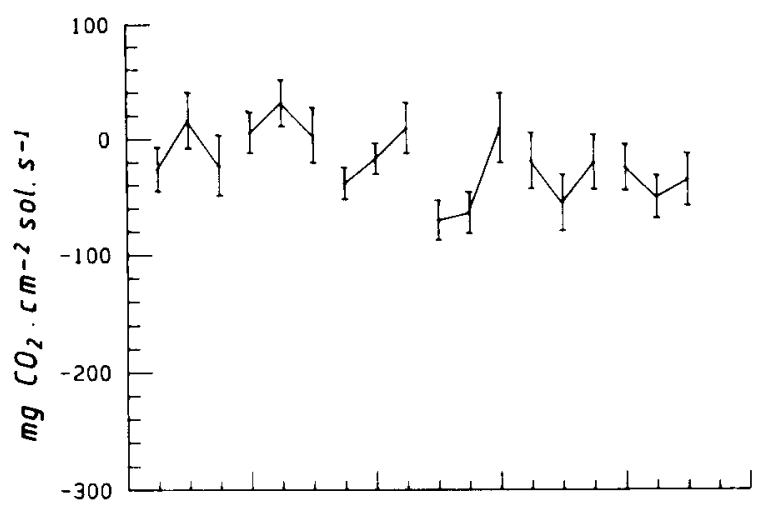

b

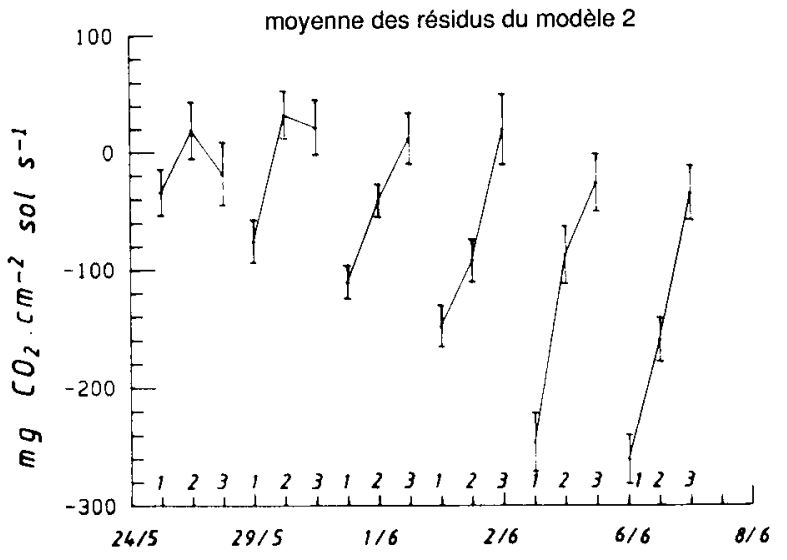

interaction jour-classe d'heure $1: 0-3 \mathrm{~h} ; 2: 3-6 \mathrm{~h} ; 3:>6 \mathrm{~h}$.

Fig 4. Analyse des interactions entre jour et heure de mesure. Les résidus des simulations de chaque jour de $\mathrm{mesure}\left(\mathrm{mg} \mathrm{CO}_{2} \cdot \mathrm{m}^{-2}\right.$ de sol. $\mathrm{s}^{-1}$ ) ont été regroupés par classe d'écart au midi solaire (classes de $3 \mathrm{~h}$ ). Les valeurs correspondant aux classes d'une même journée sont reliées entre elles par un trait continu. a: modèle 1, sans effet âge. b: modèle 2 , avec effet âge.

Tableau III. Photosynthèse maximale et efficience quantique comparées des différents modèles.

$$
\begin{aligned}
& \text { Modèle } \quad P_{\text {max }} \quad E_{i} \\
& \left(m g \mathrm{CO}_{2} \cdot m^{-2} \cdot s^{-1}\right) \quad\left(m g \mathrm{CO}_{2} \cdot \mu m \mathrm{~mol}^{-1}\right)
\end{aligned}
$$

$\begin{array}{lll}\text { Acock et al } & 1,47 & 0,0022 \\ \text { Modèle 1 } & 1,40 & 0,0016 \\ \text { Modèle 2 } & 2,01 & 0,0025\end{array}$

Il apparaît clairement que les valeurs de photosynthèse sont sous-estimées au voisinage du midi solaire; pendant le reste de la journée les résidus restent très proches de 0 . En fait, l'analyse des interactions entre jour et heure de mesure (fig 4a) met en évidence 2 journées pour lesquelles cette sous-estimation est significative : il s'agit du 1 er et du 2 juin, c'est-à-dire les 2 journées pour lesquelles les conditions de rayonnement étaient très variables; dans ces conditions, la sélection des heures de mesure pour lesquelles on peut considérer qu'il n'y a pas eu de perturbation de la mesure de rayonnement par des ombres portées devient extrêmement difficile. On ne peut donc pas clairement déterminer si ces forts résidus sont dûs à un fonctionnement différent des plantes dans ces conditions particulières, ou à un artefact dans la détermination du rayonnement incident.

\section{Comparaison entre les deux modèles}

Par rapport au modèle 1 , le modèle 2 qui introduit un effet de l'âge de la feuille sur son activité, présente une tendance à sous-estimer de façon plus importante a photosynthèse du couvert, tout au moins pour les valeurs de rayonnement élevées.

L'analyse des interactions entre jour et heure de mesure (fig 4b) fait apparaître 2 éléments complémentaires qui déterminent cette sous-estimation :

Un effet très marqué de l'heure existe pour tous les jours de mesure sans exception, et se manifeste aussi par une augmentation des résidus au voisinage du midi solaire. Compte tenu de la structure du couvert et de la répartition des feuilles, ceci correspond aux périodes pendant lesquelles les feuilles inférieures reçoivent une quantité importante de rayonnement; il semble donc que le modèle ait tendance à sous-estimer l'activité des feuilles les plus âgées, ou en d'autres termes que le paramètre $P I_{m}$ ait été sousestimé.

Une augmentation des résidus avec le temps existe aussi. Ils passent d'une valeur moyenne de -15 $\mathrm{mg}$ de $\mathrm{CO}_{2} \cdot \mathrm{m}^{-2} \cdot \mathrm{S}^{-1}$ pour les premières journées (2629 mai) à -160 pour le 8 juin; cette évolution peut aussi résulter d'une sous-estimation de l'activité des feuilles âgées, dans la mesure où, au fur et à mesure du développement de la culture, ces feuilles plus âgées prennent une importance de plus en plus grande. On en trouvera une confirmation dans la figure 5, qui présente la différence entre les 2 modèles, en fonction du niveau de rayonnement reçu par la culture. On voit très nettement se séparer 3 groupes de journées :

- d'une part, le 24 et le 26 mai, qui correspondent à des simulations réalisées à partir de mesures de surface foliaire et de structure du feuillage réalisées le 27 mai;

- d'autre part, le 6 et le 8 juin, simulées à partir de mesures de structures le 7 juin;

- enfin, du 29 mai au 2 juin, simulations pour lesquelles les données de structure du couvert ont été calculées par interpolation entre les deux dates de mesure précédentes. 


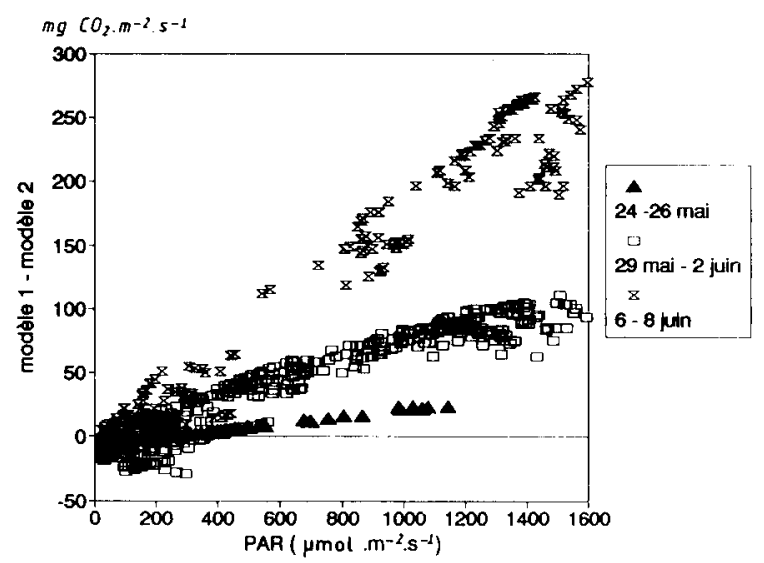

Fig 5. Ecart entre les estimations des 2 modèles $\left(\mathrm{mg} \mathrm{CO} \mathrm{CO}_{2} \bullet \mathrm{m}^{-}\right.$ 2 de sol $\left.\cdot \mathrm{s}^{-1}\right)$, en fonction du rayonnement incident $\left(\mu \mathrm{mol} \cdot \mathrm{m}^{-}\right.$ $2 \cdot \mathrm{s}^{-1}$ ), pour les différentes dates de simulation.

A l'intérieur de chacun de ces groupes, il existe une relation quasi-linéaire entre les simulations fournies par les 2 modèles. Si pour les premiers jours, la différence reste toujours négligeable, par la suite la pente de la droite devient significative : les différences les plus importantes se produisent pour les fortes valeurs de rayonnement car les échanges gazeux simulés par le modèle 2 présentent une saturation de la réponse au rayonnement plus rapide que ceux du modèle 1 . Il est également possible que cette "dérive» soit due à une mauvaise description de la structure du feuillage; toutefois dans ce cas, il serait logique de trouver les plus grands écarts pour les journées comprises entre le 29 mai et le 2 juin, pour lesquelles cette structure a été déduite par interpolation entre les deux autres périodes, et donc avec une plus grande incertitude.

\section{DISCUSSION}

\section{Simulation de résultats de la littérature}

Nous avons utilisé les modèles 1 et 2 pour simuler l'expérience présentée par Acock et al (1978), dans laquelle, par des effeuillages successifs, la contribution des feuilles de différents niveaux à la photosynthèse de la culture a été mesurée. Les résultats du modèle 1 , peu différent par ses paramètres des observations de Acock et al (tableau III), montrent une plus faible photosynthèse totale de la culture, due à une moins grande activité de la couche supérieure (fig 6).

Le modèle 2 , prenant en compte l'effet de l'âge, aboutit à une photosynthèse totale peu différente de celle mesurée par Acock et al, mais répartie différemment. La couche inférieure a une activité quasi nulle, alors que la couche supérieure réalise l'essentiel de la photosynthèse. La très faible activité calculée pour la couche inférieure est due à la prise en compte de l'âge des feuilles, puisque les résultats du modèle 1 montrent qu'il reste assez de rayonnement disponible à ce niveau pour permettre une activité photosynthétique plus importante. II ressort de cette comparaison avec les mesures de Acock et al, que le modèle 2 semble surestimer la réduction d'activité photosynthétique liée à l'âge des feuilles.

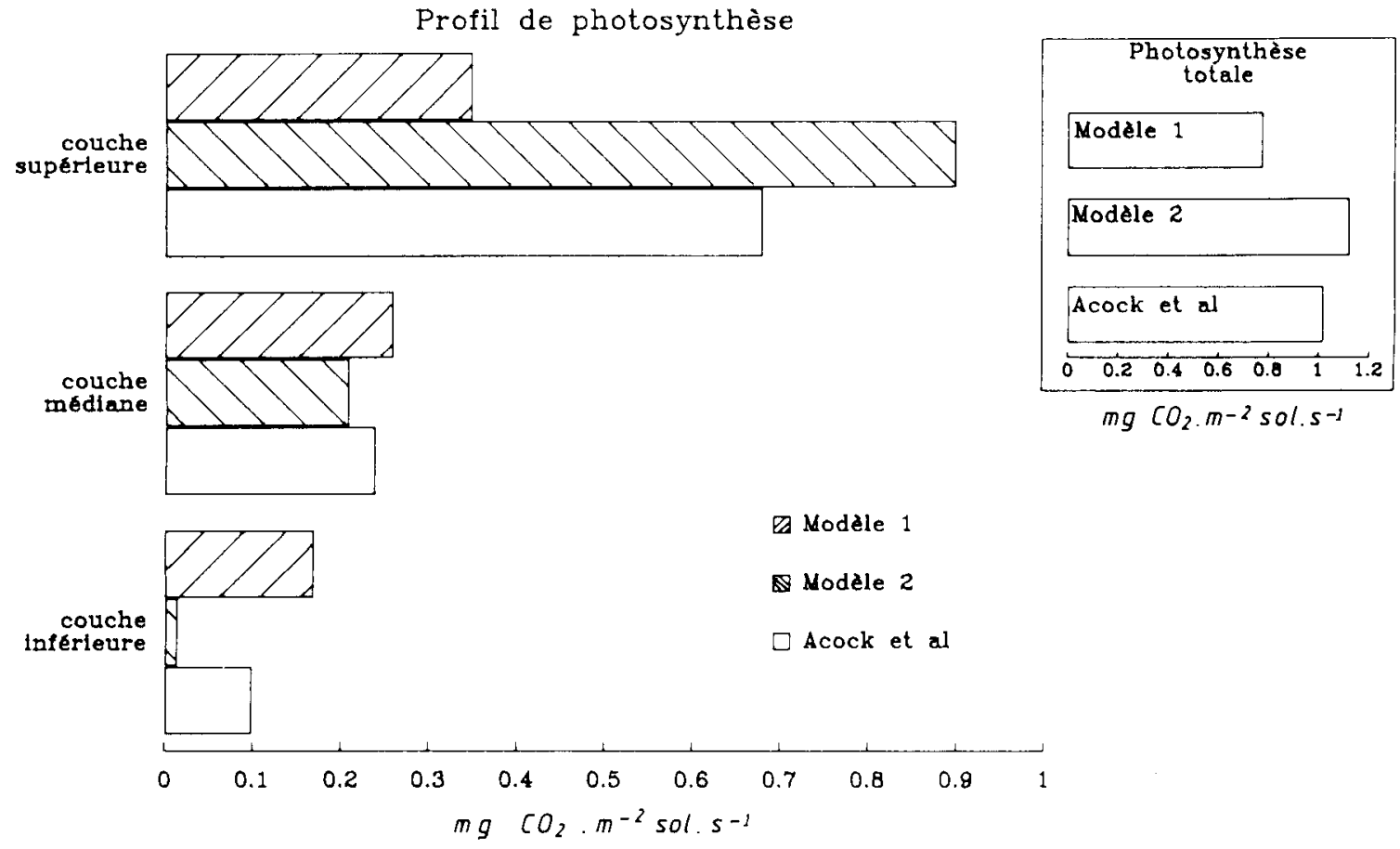

Fig 6. Comparaison des mesures de Acock et al et des résultats de simulations obtenus avec nos 2 modèles (photosynthèse brute totale et répartition par couche). 


\section{Qualité du modèle présenté}

La sous-estimation de l'activité du couvert par le modèle 2 peut être due à une sous-estimation de l'activité des feuilles les plus âgées lors des mesures instantanées qui ont servi à caler les paramètres de la fonction de photosynthèse foliaire. En effet, pour éviter de perturber durablement le fonctionnement des feuilles par une élévation de température importante, les mesures de photosynthèse maximale étaient réalisées quelques dizaines de secondes seulement après que la feuille ait été placée sous éclairement saturant; il est possible que dans ces conditions l'activité de la feuille n'ait pas atteint son maximum.' En effet plusieurs auteurs parlent de "temps d'induction" de plusieurs dizaines de minutes (Rabinowitch, 1956; Chazdon et Pearcy, 1986).

Les cartographies de photosynthèse simulée soulignent bien le rôle de la structure en rangs de couvert. Par temps clair, et pour des hauteurs moyennes de soleil (environ $1 \mathrm{~h}$ avant et après le midi solaire), les feuilles dont l'activité surfacique est importante peuvent être situées jusqu'au tiers inférieur de la plante, en bordure des allées (fig 7a). Cette activité est essentiellement due à une exposition au rayonnement direct. La détermination de la distribution d'inclinaison de ces feuilles est donc importante. Nos observations montrent que l'inclinaison prédominante de ces feuilles se situe entre 36 et $54^{\circ}$ (Tchamitchian, 1990). Leur surface est donc quasiment orthogonale au rayonnement direct qui les atteint à ce moment. Dans de telles conditions, les distributions d'inclinaisons uniformes et sphériques généralement adoptées (Goudriaan, 1989; Nederhoff et al, 1989) conduisent donc à sous-estimer l'activité de ces feuilles de bordures.

La réduction de photosynthèse totale observée autour du midi solaire pour des rangs orientés nord-sud (Gijzen et Goudriaan, 1989), que nous simulons aussi, s'explique fort bien par la réduction de la surface foliaire soumise à un fort rayonnement. Les parties de la plante dont l'activité spécifique est forte sont moins étendues que pour des hauteurs intermédiaires (fig $7 b$ ).

\section{CONCLUSION}

Le modèle que nous avons présenté montre une bonne adéquation aux mesures de validations que nous avons effectuées. La description proba-
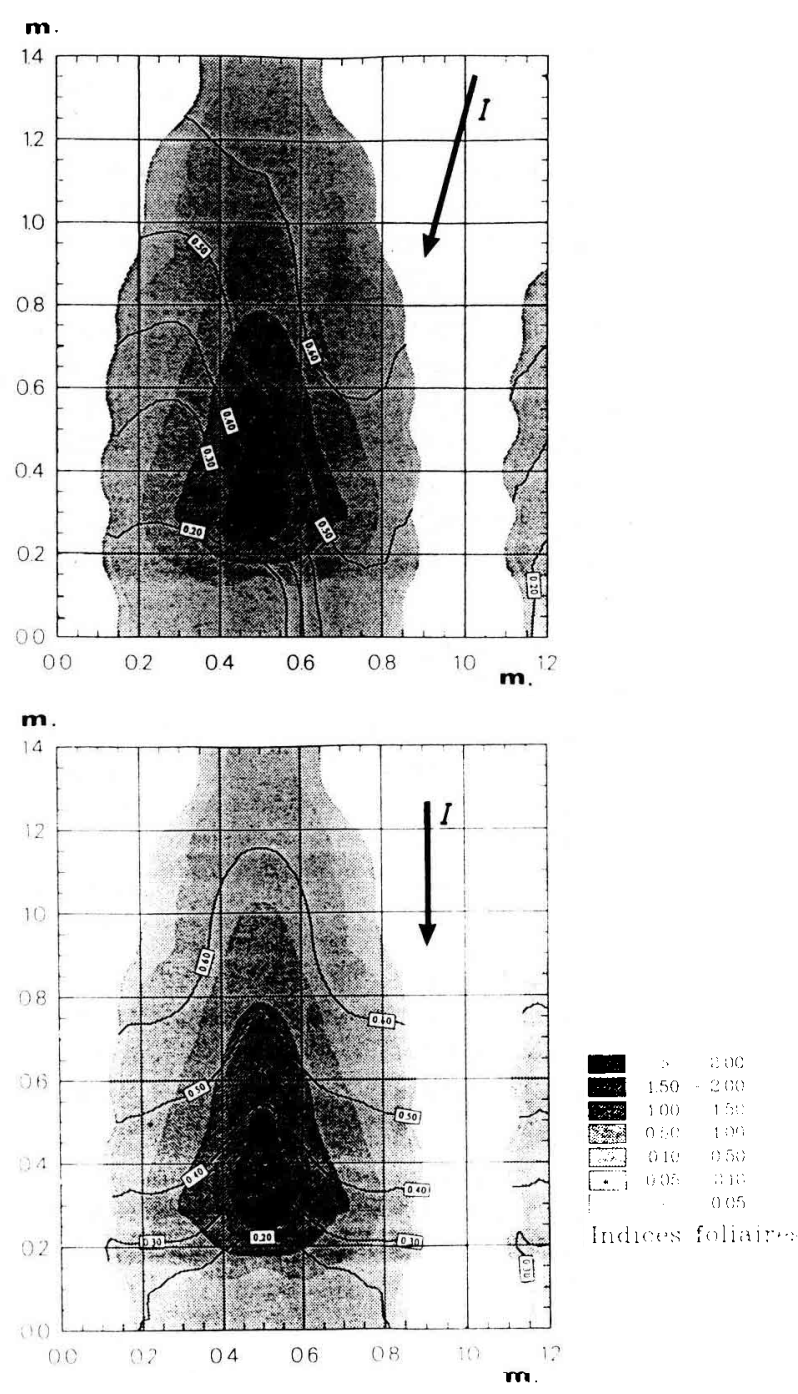

Fig 7. Cartographie de l'activité photosynthétique du feuillage en $\mathrm{mg}$ de $\mathrm{CO}_{2} \cdot \mathrm{m}^{-2}$ de feuille $\mathrm{s}^{-1}$. Coupe verticale dans un plan perpendiculaire à l'axe du rang. Simulation réa-

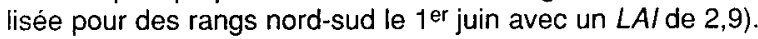
l: direction des rayons solaires; a: $11 \mathrm{~h}$ (temps solaire vrai); b: $12 \mathrm{~h}$ (tsv). Les niveaux de gris représentent les densités de surface foliaire.

biliste de la culture lui permet de s'adapter facilement aux divers types de conduites culturales (rangs simples ou doubles, variations des distances entre plantes et entre rangs). II fournit une cartographie de l'activité photosynthétique de la culture qui pourra être utilisée, après validation complète, pour simuler l'influence immédiate de pratiques culturales modifiant le port de la plante et la répartition de la surface foliaire.

\section{REMERCIEMENTS}

Ce travail a été en partie financé par le Conseil régional Provence-Alpes-Côte d'Azur. 


\section{RÉFÉRENCES}

Acock B, Charles-Edwards DA, Fitter DJ, Hand DW, Ludwig LJ, Warren-Wilson J, Withers AC (1978) The contribution of leaves from different levels within a tomato crop to canopy net photosynthesis: An experimental examination of two canopy models. J Exp Bot 29, 815-827

Acock B, Hand DW, Thornley JHM, Warren-Wilson J (1976) Photosynthesis in stands of green pepper. An application of empirical and mechanistic models to controlled-environment data. Ann Bot 40, 1293 1307

Allen LH Jr (1974) Model of light penetration into a wide row crop. Agron J 66, 41-47

Bonhomme R, Varlet-Grancher C (1978) Estimation of the gramineous crop geometry by plant profiles including leaf width variations. Photosynthetica 12 , 193-196

Charles-Edwards DA, Thorpe MR (1976) Interception of diffuse and direct-beam radiation by a hedgerow apple orchard. Ann Bot 40, 603-613

Chartier P (1969) Assimilation nette d'une culture couvrante. 2. La réponse de l'unité de surface de feuille. Ann Physiol Vég 11, 221-263

Chazdon RL, Pearcy RW (1986) Photosynthetic responses to light variation in rainforest species. I. Induction under constant and fluctuating light conditions. Oecologia 69, 517-523

Cohen S, Fuchs M (1987) The distribution of leaf area, radiation, photosynthesis and transpiration in a shamouti orange orchard. I. Leaf area and radiation. Agric For Meteorol 40, 123-144

Daudet FA (1987) Un système simple pour la mesure in situ des échanges gazeux de couverts végétaux de quelques mètres carrés de surface foliaire. Agronomie 7, 133-139

Gary C (1989) Temperature and time-course of the carbon balance of vegetative tomato plants during prolonged darkness: examination of a method of estimating maintenance respiration. Ann Bot 63, 449-458
Gijzen H, Goudriaan J (1989) A flexible and explanatory model of light distribution and photosynthesis in row crops. Agric For Meteorol 48, 1-20

Goudriaan J (1989) The bare bones of leaf-angle distribution in radiation models for canopy photosynthesis and energy exchange. Agric For Meteorol 43, 155-169

Iwakiri S, Inayama M (1974) Studies on the canopy photosynthesis of the horticultural crops in controlled environment. Geometrical structures and penetration of direct solar radiation into cucumber canopy. J Agric Meteorol (Tokyo) 30, 1-9

Jackson JE, Palmer JW (1979) A simple model of light transmission and interception by discontinuous canopies. Ann Bot 44, 381-383

Lemeur R, Blad BL (1974) A critical review of light models for estimating the shortwave radiation regime of plant canopies. Agric Meteorol 14, 255-286

Nederhoff E, Gijzen H, Vegter J (1989) A dynamic simulation model for greenhouse cucumber (Cucumis sativus $\mathrm{L}$ ): validation of the submodel for crop photosynthesis. Acta Hortic 248, 255-263

Rabinowitch El (1956) Photosynthesis and related processes. Vol II Part 2. Intersci Publ, New York

Sinoquet H (1989) Modélisation de l'interception des rayonnements solaires dans une culture en rang. I. Aspects théoriques. Agronomie 9, 125-135

Tchamitchian M (1990) Photosynthèse d'une culture de tomates sous serre : mise au point et validation d'un modèle analytique. Thèse de DDI, Institut Polytechnique de Toulouse, $98 \mathrm{p}$

Thorpe MR, Saugier B, Auger S, Berger A, Methy M (1978) Photosynthesis and transpiration of an isolated tree: model and validation. Plant Cell Environ 1, 269-277

Van Bavel CHM (1975) A behavioral equation for leaf carbon dioxide assimilation and a test of its validity. Photosynthetica 9, 165-176

Whitfield DM (1986) A simple model of light penetration into row crops. Agric For Meteorol 36, 297-315 